\title{
Screening in graphene antidot lattices
}

\author{
M. H. Schultz, ${ }^{1}$ A. P. Jauho, ${ }^{1,2}$ and T. G. Pedersen ${ }^{3}$ \\ ${ }^{1}$ Department of Micro and Nanotechnology, DTU Nanotech, Bldg. 345B, \\ Technical University of Denmark, DK-2800 Kongens Lyngby, Denmark \\ ${ }^{2}$ Department of Applied Physics, Helsinki University of Technology, P.O.Box 1100, FI-02015 TKK, Finland \\ ${ }^{3}$ Department of Physics and Nanotechnology, Aalborg University, DK-9220 Aalborg East, Denmark
}

(Dated: July 1, 2018)

\begin{abstract}
We compute the dynamical polarization function for a graphene antidot lattice in the randomphase approximation. The computed polarization functions display a much more complicated structure than what is found for pristine graphene (even when evaluated beyond the Dirac-cone approximation); this reflects the miniband structure and the associated van Hove singularities of the antidot lattice. The polarization functions depend on the azimuthal angle of the q-vector. We develop approximations to ease the numerical work, and critically evaluate the performance of the various schemes. We also compute the plasmon dispersion law, and find an approximate square-root dependence with a suppressed plasmon frequency as compared to doped graphene. The plasmon dispersion is nearly isotropic, and the developed approximation schemes agree well with the full calculation.
\end{abstract}

\section{INTRODUCTION}

Graphene Antidot Lattices (GALs) have been promoted as a flexible platform for creating a tunable band gap thereby possibly allowing the realization of a number of technological applications ${ }^{1}$. In its basic form the GAL consists of a graphene sheet with a periodic nanometer scale perforation. Alternatively, GALs can be viewed as a special realization of a superlattice imposed on pristine graphene. Such a superlattice can be fabricated by a variety of technologies, for example by a periodic chemical modification, by selective adsorption of atoms or molecules on graphene, by periodic arrangement of electrostatic gates, or by an intrinsic or extrinsic regular corrugation. There is already a substantial literature on this general topic (see, e.g., Refs. [2 7]); here we focus on the antidot lattice case. Theoretically the properties of triangular GALs have been examined already quite substantially (e.g., optical properties $\stackrel{\underline{8}}{ }$, excitons $\stackrel{9}{=}$, electronic properties 10,11 , electron-phonon coupling 12,13 , detection of edge states $\frac{14}{4}$, or details of band gap scaling $\frac{15}{5}$ ). Most importantly, the experimental techniques for fabricating GALs have evolved rapidly $\underline{\underline{16}} \stackrel{-19}{=}$, presently reaching lattice constants down to a few tens of nanometers, where many of the interesting quantum mechanical effects predicted by theory should become detectable. Of particular interest are transport measurements, which we expect soon to be available.

Most of the early works on GALs have focused on single-particle effects, such as the miniband structure, sometimes supported by $a b$ initio calculations using density-functional theory (DFT), or by some other less accurate but computationally more effective method, such as the density-functional based tightbinding (DFTB) $\stackrel{20}{2}$. However, it is necessary also to consider electron-electron interactions since these affect many physical properties, such as the charge carriers' interaction with light or with lattice vibrations, dielectric screening and plasmons, or the transport properties.
The dynamical polarization function is a central object in these considerations, and it has been a topic of wide interest. In the literature expressions relevant for the polarization function of graphene, evaluated in the random phase approximation, can be found already from the "pre-graphene" era (see, e.g., Refs. 21,22]). More recently, thorough studies of the polarization function in the Dirac-cone approximation have been reported in Refs. 23, 24], but only very recently Stauber et al. $\stackrel{25,26}{ }$ gave similar results for full graphene dispersion at finite chemical potential. It should be noted that the accuracy of the RPA for pristine graphene is a highly nontrivial issue, see, e.g., Ref. [27], and references cited therein. Many of the complications encountered in these studies originate from the high-momentum cutoff needed in the Coulomb interaction, when extending the discussion beyond RPA, and are specific to the Dirac cone dispersion law. Here, we consider the full GAL dispersion with a gap, and believe that the RPA is a justifiable starting point. Our calculations are conceptually straightforward: indeed the formal expressions for the Lindhard function for a general tight-binding model (which will be our underlying antidot lattice Hamiltonian) are standard textbook material (see, e.g., Sect. 8.5 in Ref. 28]). Some complications inevitably arise because the antidot lattice unit cells contain tens or hundreds of atoms, in contrast to just two in pristine graphene. Analytic expressions can hardly be expected for such a complicated system, and much of our effort goes into examining whether simpler yet reasonably accurate models can reproduce the results of the full numerical calculations. The GALs can be realized with many different lattice symmetries, each of which may have some interesting physics of its own (see, e.g. Refs. 11,29,30]): whether a gap arises in the energy spectrum depends in a sensitive way on the symmetry of the antidot lattice, and on the orientation of the antidot lattice with respect to the underlying graphene lattice. Here we examine one particular GAL (with a triangular lattice symmetry) in detail as a demonstration 
of the theoretical methodology, and defer the discussion of other lattice symmetries until experiments performed on well-characterized samples become available.

This paper is organized as follows. In Sect. II we briefly introduce the basic models used in this work: the tightbinding representation of the antidot lattice states, and a simplified model, the "gapped graphene". In Sect. III we give both analytical and numerical results for the polarization function, address certain issues in the numerical computation, and present a comparison between the various approximation schemes. Sect. [V] addresses the plasmonic properties of GALs, and we end with a brief conclusion.

\section{THE MODELS}

\section{A. Antidot lattice energy spectrum}

We use a tight-binding model to compute the band structure of the GALs. The wave function is written as

$$
\psi_{n \mathbf{k}}(\mathbf{r})=\frac{1}{\sqrt{N}} \sum_{\mathbf{R}} e^{i \mathbf{k} \cdot \mathbf{R}} \sum_{j=1}^{M} c_{n \mathbf{k}}^{j} \phi\left(\mathbf{r}-\mathbf{R}-\mathbf{d}_{j}\right),
$$

where $\phi(\mathbf{r})$ is the normalized atomic wave function of the $2 p_{z}$ orbital of carbon, and the $j$-sum runs over the atoms in the unit cell of the antidot lattice. $\mathbf{R}$ runs over the unit cells in the antidot lattice. The coefficients $c_{n \mathbf{k}}^{j}$ and the eigenenergies $\epsilon_{n \mathbf{k}}$ are then obtained from the $M \times M$ eigenvalue problem, for each value of the wave vector $\mathbf{k}$ in the 1st Brillouin zone of the antidot lattice. Each GAL is characterized by a pair of indices $\{L, R\}$, where the $L$ index gives the side length of the hexagonal unit cell, and the $R$ index is the (approximate) radius of the hole, in units of the graphene lattice constant $\sqrt{3} a$, where $a=1.42 \AA$ is the C-C distance ${ }^{1}$. Figure 1 shows an example of a typical GAL band structure.

\section{B. Gapped graphene}

The gapped graphene model is a phenomenological model used to describe band structures with a gap. Consider first the pristine graphene dispersion $\epsilon_{n \mathbf{k}}^{0}=$ $n t|\phi(\mathbf{k})|$, where $t=3.03 \mathrm{eV}$ is the hopping integral, $n= \pm 1$ and

$$
\phi(\mathbf{k})=e^{i \mathbf{k} \cdot \boldsymbol{\delta}_{1}}+e^{i \mathbf{k} \cdot \boldsymbol{\delta}_{2}}+e^{i \mathbf{k} \cdot \boldsymbol{\delta}_{\mathbf{3}}},
$$

where $\boldsymbol{\delta}_{i}$ are the three neighbors of the reference atom at the origin $\left(\boldsymbol{\delta}_{1}=a / 2(-1, \sqrt{3}), \boldsymbol{\delta}_{2}=a / 2(-1,-\sqrt{3})\right.$, $\left.\boldsymbol{\delta}_{3}=a(1,0)\right)$. Next, introduce a shift $\pm \Delta$ on the onsiteenergies for the carbon atoms on the $A$ - and $B$ - sublattices, respectively. This gives rise to a new spectrum,

$$
\epsilon_{n \mathbf{k}}=n \sqrt{\Delta^{2}+t^{2}\left|\phi_{\mathbf{k}}\right|^{2}}
$$

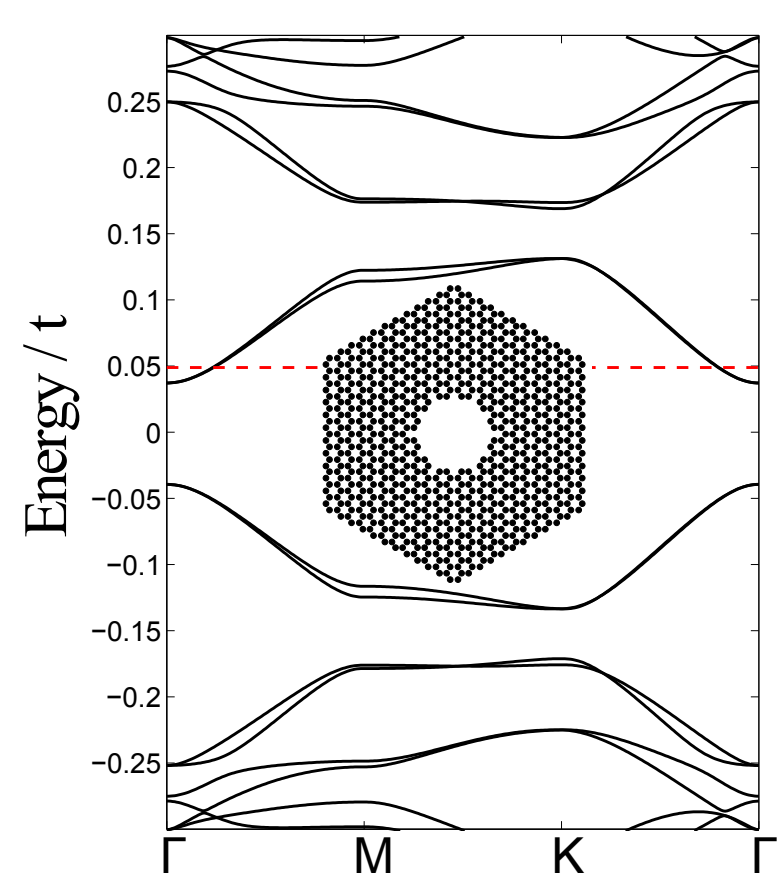

FIG. 1: (Color online) Band structure of a $\{12,3\}$ GAL with a circular antidot with an armchair edge. The inset shows the atomistic configuration of the unit cell. The dashed line indicates the value of the chemical potential, $\mu=0.05 t$ ( $t$ is the hopping integral), used in most of the subsequent calculations.

where $n= \pm 1$, and corresponding eigenvectors

$$
\mathbf{v}_{n \mathbf{k}}=\frac{1}{\sqrt{2}}\left(\frac{\frac{-n t \phi_{\mathbf{k}}}{\sqrt{\left|\epsilon_{n \mathbf{k}}\right|\left(\left|\epsilon_{n \mathbf{k}}\right|-n \Delta\right)}}}{\sqrt{\frac{\left|\epsilon_{n \mathbf{k}}\right|-n \Delta}{\left|\epsilon_{n \mathbf{k}}\right|}}}\right) .
$$

Since $\phi(\mathbf{k})$ vanishes at the Dirac points $K, K^{\prime}$, the energy gap is $E_{g}=\epsilon_{+, \mathbf{K}}-\epsilon_{-, \mathbf{K}}=2|\Delta|$, and $\Delta$ can be chosen so that the spectrum fits the gap of a given antidot lattice spectrum. The gapped graphene can also be viewed as a minimal perturbation of the pristine graphene, e.g., in the case of very small antidots compared to the full unit cell. If, moreover, the chemical potential is close to the band edge so that the low-energy part of the band structure dominates, we expect the gapped graphene model to be accurate. Below we give explicit examples of what "low-energy" means in practice.

\section{POLARIZATION FUNCTION}

\section{A. Analytical results}

The polarization function is evaluated from the density-density response function for noninteracting elec- 
trons:

$$
\begin{aligned}
\chi_{0}^{r}(\mathbf{q}, \omega)= & \frac{2}{(2 \pi)^{2}} \sum_{n n^{\prime} \mathbf{k}^{\prime}} \int_{1 . \mathrm{BZ}} d \mathbf{k}\left|\left\langle n \mathbf{k}\left|e^{-i \mathbf{q} \cdot \mathbf{r}}\right| n^{\prime} \mathbf{k}^{\prime}\right\rangle\right|^{2} \\
& \times \frac{n_{F}\left(\epsilon_{n \mathbf{k}}\right)-n_{F}\left(\epsilon_{n^{\prime} \mathbf{k}^{\prime}}\right)}{\epsilon_{n \mathbf{k}}-\epsilon_{n^{\prime} \mathbf{k}^{\prime}}+\omega+i \eta} .
\end{aligned}
$$

Here, $n_{F}$ is the Fermi-Dirac distribution, which we evaluate at a fixed temperature of $k_{B} T=0.01 t$ throughout. In writing Eq. (5) we have assumed that local field effects can be neglected, i.e., the relevant wave vectors $q$ are small compared to the reciprocal lattice vectors $G \propto 1 / L$. This allows us to work with a scalar dielectric function, rather than the more general object defined in the $\mathbf{G}, \mathbf{G}^{\prime}$-space. The price is that certain collective intervalley modes will not be included (see, e.g., Ref. [31]).

In addition to the energy spectrum one also needs to evaluate the matrix element. In the following we list the required matrix element for the various cases of interest, including some well-known results for completeness.

\section{Pristine graphene}

The matrix element is 25

$$
\begin{aligned}
& \left|\left\langle n \mathbf{k}\left|e^{-i \mathbf{q} \cdot \mathbf{r}}\right| n^{\prime} \mathbf{k}^{\prime}\right\rangle\right|^{2}=\delta_{\mathbf{k}^{\prime}, \mathbf{k}+\mathbf{q}} \\
& \times \frac{1}{2}\left(1+n n^{\prime} \operatorname{Re}\left[e^{i \mathbf{q} \cdot \delta_{3}} \frac{\phi_{\mathbf{k}}}{\left|\phi_{\mathbf{k}}\right|} \frac{\phi_{\mathbf{k}+\mathbf{q}}^{*}}{\left|\phi_{\mathbf{k}+\mathbf{q}}\right|}\right]\right),
\end{aligned}
$$

where the various quantities are defined in Eq.(2).

\section{Dirac cone}

With the spectrum linearized in the vicinity of the Dirac points,

$$
\epsilon_{n \mathbf{k}}^{0} \approx n \frac{3 a t}{2} k,
$$

Eq.(6) simplifies, and one finds ${ }^{23,24}$

$$
\left|\left\langle n \mathbf{k}\left|e^{-i \mathbf{q} \cdot \mathbf{r}}\right| n^{\prime} \mathbf{k}^{\prime}\right\rangle\right|^{2}=\delta_{\mathbf{k}^{\prime}, \mathbf{k}+\mathbf{q}} \frac{1}{2}\left[1+n n^{\prime} \cos \left(\theta_{\mathbf{k}, \mathbf{k}+\mathbf{q}}\right)\right],
$$

where $\theta_{\mathbf{k}, \mathbf{k}+\mathbf{q}}$ is the angle between $\mathbf{k}$ and $\mathbf{k}+\mathbf{q}$.

\section{Gapped graphene} finds

Using the eigenenergies (3) and eigenvectors (44) one

$$
\begin{aligned}
& \left|\left\langle n \mathbf{k}\left|e^{-i \mathbf{q} \cdot \mathbf{r}}\right| n^{\prime} \mathbf{k}^{\prime}\right\rangle\right|^{2}=\delta_{\mathbf{k}^{\prime}, \mathbf{k}+\mathbf{q}} \\
& \times \frac{1}{2}\left(\frac{t^{4}\left|\phi_{\mathbf{k}}\right|^{2}\left|\phi_{\mathbf{k}^{\prime}}\right|^{2}+\left(\left|\epsilon_{n \mathbf{k}}\right|-n \Delta\right)^{2}\left(\left|\epsilon_{n \mathbf{k}^{\prime}}\right|-n^{\prime} \Delta\right)^{2}}{2\left|\epsilon_{n \mathbf{k}}\right|\left(\left|\epsilon_{n \mathbf{k}}\right|-n \Delta\right)\left|\epsilon_{n \mathbf{k}^{\prime}}\right|\left(\left|\epsilon_{n \mathbf{k}^{\prime}}\right|-n^{\prime} \Delta\right)}\right. \\
& \left.+n n^{\prime} \operatorname{Re}\left[e^{i \mathbf{q} \cdot \boldsymbol{\delta}_{3}} \frac{t^{2} \phi_{\mathbf{k}} \phi_{\mathbf{k}^{\prime}}^{*}}{\left|\epsilon_{n \mathbf{k}}\right|\left|\epsilon_{n \mathbf{k}^{\prime}}\right|}\right]\right)
\end{aligned}
$$

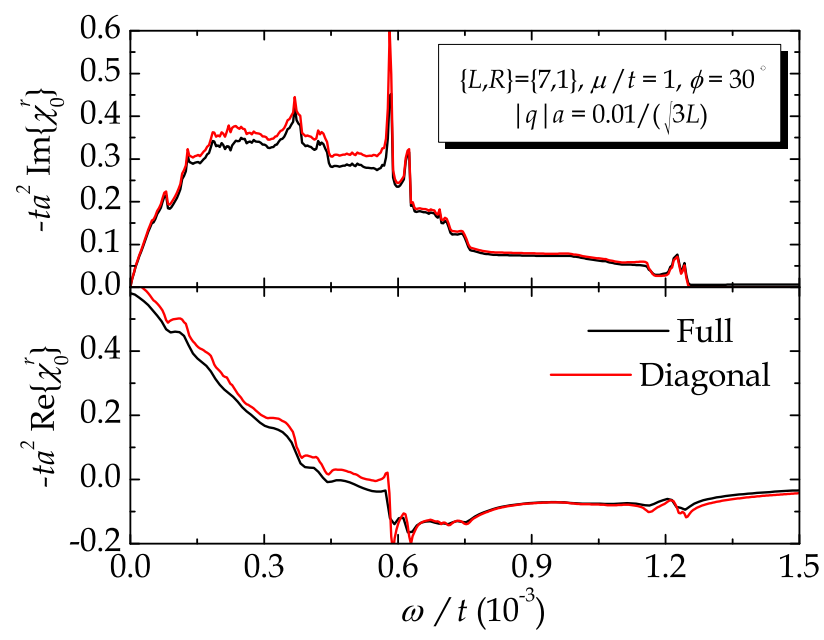

FIG. 2: (Color online) $\operatorname{Im} \chi_{0}^{r}(\mathbf{q}, \omega)$ (top panel), and $\operatorname{Re} \chi_{0}^{r}(\mathbf{q}, \omega)$ (bottom panel) as a function of $\omega / t$ for a $\{7,1\}$ GAL. $\phi$ defines the azimuthal angle of the q-vector. The curve labeled "full" is evaluated using the full expression Eq. (10), while the curve labeled "diagonal" employs the approximation Eq. (11).

\section{Antidot lattice}

Using the tight-binding wave functions (11) the matrix element becomes

$$
\left\langle n \mathbf{k}\left|e^{-i \mathbf{q} \cdot \mathbf{r}}\right| n^{\prime} \mathbf{k}^{\prime}\right\rangle=\delta_{\mathbf{k}^{\prime}, \mathbf{k}+\mathbf{q}} \sum_{j=1}^{M}\left(c_{n \mathbf{k}}^{j}\right)^{*} c_{n^{\prime} \mathbf{k}+\mathbf{q}}^{j} e^{-i \mathbf{q} \cdot \mathbf{d}_{j}} .
$$

It is readily verified that the general expression (10) reproduces the standard results (i.e., set $M=2, \mathbf{d}_{1}=\mathbf{0}$, and $\mathbf{d}_{2}=\boldsymbol{\delta}_{3}$ for pristine graphene). The challenge in the numerical applications is that for each q-point a large number of terms needs to be considered, and that a full Brillouin zone summation is required for every term. Below we discuss approximate methods of how to bypass this difficulty.

\section{B. Numerical results}

\section{The limit $q L \ll 1$}

As remarked above, in this work we do not consider local field effects, i.e., we restrict our discussion to wavevectors that are small compared to the reciprocal lattice vectors, $q L \ll 1$ (a similar restriction was used in Ref. 25]). We may exploit this restriction further by noting that the vectors $\mathbf{d}_{j}$ entering the matrix element (10) satisfy $d_{j}<L$, and therefore the phase-factor can be approximated as $\left|\exp \left(-i \mathbf{q} \cdot \mathbf{d}_{j}\right)\right| \simeq 1$. Further, since the wave vectors $\mathbf{q}$ in general are small, for most of the $\mathbf{k}$ vectors entering the Brillouin zone summations the eigenvectors corresponding to $\mathbf{k}$ and $\mathbf{k}+\mathbf{q}$ do not differ much, and therefore we can appeal to the orthonormality (in 


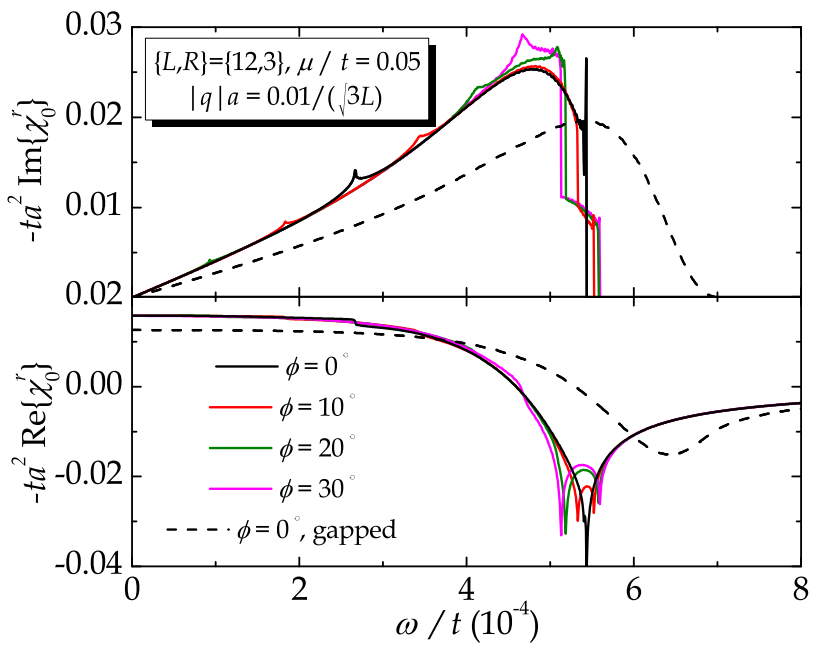

FIG. 3: (Color online) $\operatorname{Im} \chi_{0}^{r}(\mathbf{q}, \omega)$ (top panel) and $\operatorname{Re} \chi_{0}^{r}(\mathbf{q}, \omega)$ (bottom panel) for the $\{12,3\}$ GAL as a function of $\omega / t$, at chemical potential $\mu / t=0.05$. The solid lines give the polarization function (using the approximation (11)) for various azimuthal angles $\phi$ while the dashed line gives the gapped graphene result.

the band indices $n, n^{\prime}$ ) of the eigenvectors, and thereby obtain the final approximation for the matrix elements:

$$
\left\langle n \mathbf{k}\left|e^{-i \mathbf{q} \cdot \mathbf{r}}\right| n^{\prime} \mathbf{k}^{\prime}\right\rangle \simeq \delta_{\mathbf{k}^{\prime}, \mathbf{k}+\mathbf{q}} \delta_{n, n^{\prime}}
$$

We emphasize that since the limits of validity of the above expression are not rigorously given, one must carefully check its reliability in each case. We have performed a large number of such numerical tests, and representative results for a typical worst case scenario are given in Fig. 2. The results for the imaginary part, Fig. 2 (top panel) agree qualitatively well: all major features are reproduced. For the large $\omega$-limit, $\omega / t>1.25$ in Fig. 2 , a small systematic difference is observed (barely observable within the resolution of the figure); we have not been able to pinpoint the reason for this tiny difference. The results for the real part, Fig. 2 (bottom panel), were obtained by performing a Kramers-Kronig transformation of the corresponding imaginary part. Here we see perhaps an even better agreement between the full numerics and the approximate result. In general, the matrix element appears to be a smooth function of $\mathbf{q}$, which does not introduce additional sharp features in the polarization function. A particularly interesting observation is that the high-energy tails of the $\operatorname{Re} \chi_{0}^{r}$, which are important in the evaluation of the plasmon spectrum, are in near quantitative agreement, and we shall exploit this fact in Sect. IV where we analyze the plasmon dispersion laws.

\section{Brillouin-zone summation}

The integrals over the Brillouin zone were carried out with an improved triangle $\operatorname{method}^{32}$, see the Appendix of

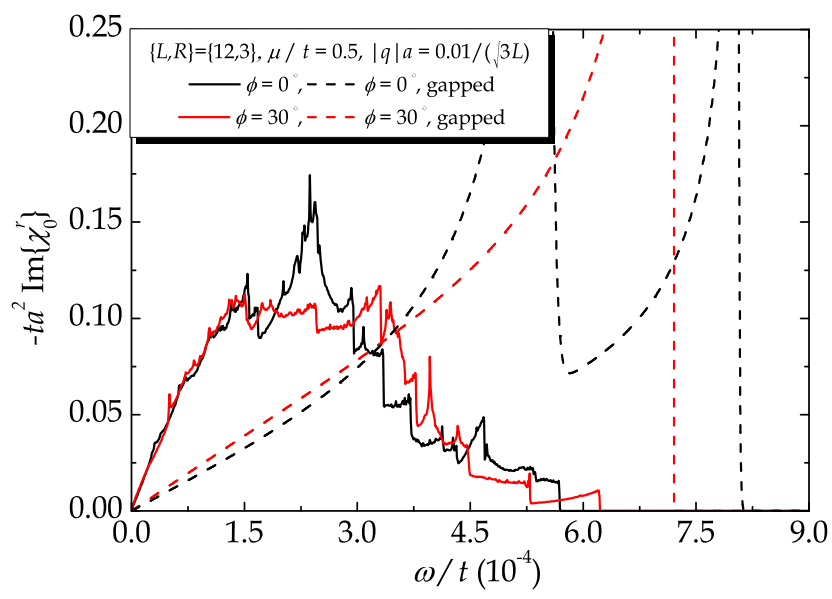

FIG. 4: (Color online) Imaginary part of the polarization function for the $\{12,3\}$ GAL as a function of $\omega / t$, at chemical potential $\mu / t=0.5$. The solid lines give the full polarization function (using the approximation (11)) for various azimuthal angles $\phi$ while the dashed line gives the corresponding gapped graphene results.

Ref. [8] for details of our specific implementation. Very briefly, the method consists of generating a grid of $\mathbf{k}$ points, and writing the BZ-integrals as sums over triangles spanned by the grid points, and performing an analytic integration within each triangle using a linear approximation for the integrand. To evaluate the integrand, the $M \times M$-eigenvalue and -vector problem must be solved for each $\mathbf{k}$ and $\mathbf{k}+\mathbf{q}$ pair. By checking first that the Fermi-function difference in (5) is nonzero, as well as that the delta-function has a zero, a certain fraction of the eigenvalue problems could be eliminated. Fortunately, the method is rapidly converging as the $\mathbf{k}$-mesh is made denser, as could be verified by bench-marking it against cases where analytic results are available, e.g., the Dirac cone dispersion. Typically we used $\simeq 5000$ $\mathbf{k}$-points in the mesh. In our calculations we always evaluate the imaginary part of Eq.(5) first, and then compute the real part by a Kramers-Kronig transformation.

\section{An example: The $\{12,3\}-G A L$}

Figure 3 gives the results for the imaginary and real part of the polarization function for the $\{12,3\}$ GAL of Fig. 1, at a relatively low chemical potential, $\mu / t=0.05$. We have chosen to study this particular GAL in detail, and it is relevant to inquire how generic our results are. Of special interest is the effect of the shape and the edge of the antidot, for example whether the edge is of the armchair or of the zigzag type. It is well-known that non-dispersive zero-energy states may occur for certain structures (see, e.g., the examples shown in Refs. [11,20)], which might lead to characteristic features in $\chi_{0}^{r}(\mathbf{q}, \omega)$. Not much is known of the robustness of these edge states against disorder, or other edge reconstructions; see how- 
ever a recent related study by $\mathrm{Li}$ et $\mathrm{a}^{3 \mathrm{33}}$. A full study of these effects is beyond the scope of the present paper, where we want to illustrate the basic features of GALs with a relatively simple band structure, as the one illustrated in Fig. 1. Another attractive feature of the chosen example is that the triangular GAL obeys a simple scaling law for the band gap ${ }^{\underline{1}}$, which implies that our results are relevant also for larger structures, for which full numerical calculations would be extremely challenging.

It is interesting to compare these results with those pertaining to pristine graphene $e^{25,26}$. A certain overall qualitative similarity persists. The imaginary part increases as a function of the frequency, and after reaching its maximum value it drops rapidly to zero. Superposed are certain sharp features: these are related to the van Hove singularities occurring at the band edges. Since there are many more bands in the GALs in a given energy range than there are for pristine graphene, it is not surprising that the number of sharp peaks is larger for GALs. A similar qualitative resemblance can be seen for the real part of the polarization function: at low energies the real part plunges deeply (passing zero), whereafter it asymptotically approaches zero from the negative side. Again, the GAL exhibits much more fine structure.

Also shown in Fig. 3 are the results for the gapped graphene model. We observe that the results depend on the azimuthal angle when the full GAL dispersion is used, whereas the gapped graphene model does now show such dependence. The overall shape of the gapped graphene model mimics fairly well the GAL results, but is obviously much smoother since the gapped graphene model with its only two bands cannot reflect the van Hove structure of the full GAL dispersion.

While the gapped graphene model performs reasonably well at low chemical potentials, as expected, it fails even qualitatively at higher $\mu$. An example is given in Fig.(4), where we show the imaginary part of the polarization function for $\mu / t=0.5$, and, as can be seen, there is only a token of resemblance between these results. The situation is even worse for the real part (not shown). We conclude that if one is interested in the fine details of the polarization function, the gapped graphene model is reliable only at very low chemical potentials, and even there caution should be exercised.

\section{PLASMONS}

We next examine the undamped plasmon modes for the $\{12,3\}$ GAL. As usual, the modes $\omega(\mathbf{q})$ are identified as solutions to

$$
1-W(\mathbf{q}) \operatorname{Re} \chi_{0}^{r}(\mathbf{q}, \omega(\mathbf{q}))=0,
$$

where $W(\mathbf{q})$ is the Coulomb interaction. We solve (12) numerically: for a fixed $\mathbf{q}$ we find the $\omega_{p}(\mathbf{q})$ 's which cause Eq.(12) to vanish, and use $\operatorname{Im} \chi\left(\mathbf{q}, \omega_{p}(\mathbf{q})\right)=0$ as a criterion to identify the undamped modes. In all cases considered the solution was unique. We shall compare the nu-

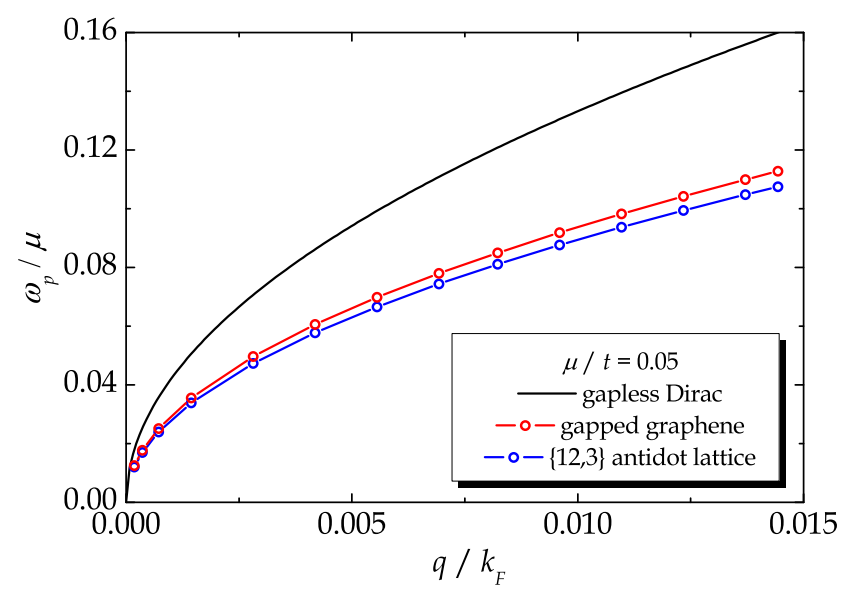

FIG. 5: (Color online) Plasmon dispersion for $\mu / t=0.05$.

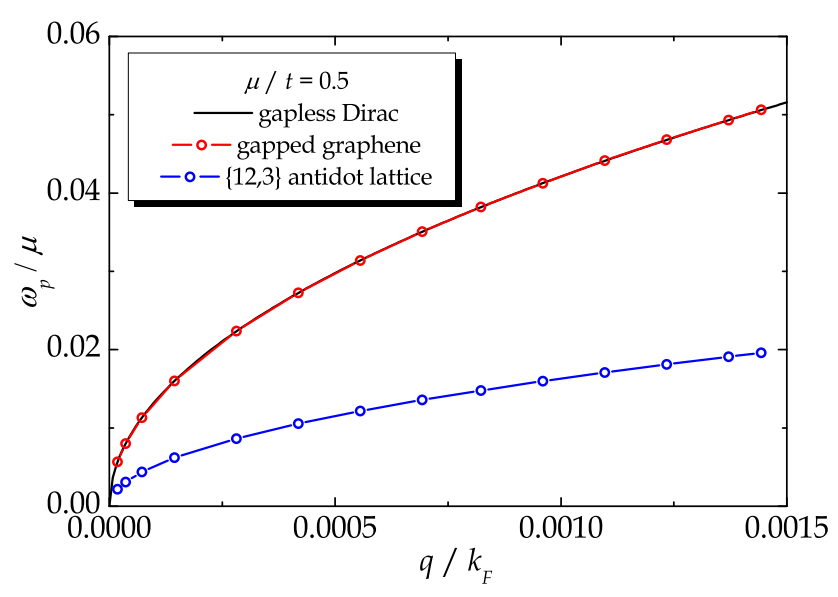

FIG. 6: (Color online) Plasmon dispersion for $\mu / t=0.5$.

merically found GAL plasmons to the well-known dispersions for pristine graphene $\frac{23}{\underline{ }} \omega_{\mathrm{gr}}$, and gapped graphene ${ }^{34}$ $\omega_{\mathrm{g}-\mathrm{gr}}$ :

$$
\omega_{\mathrm{gr}} / \mu=C \sqrt{\frac{q}{k_{F}}}, \quad \omega_{\mathrm{g}-\mathrm{gr}} / \mu=C \sqrt{1-\frac{\Delta^{2}}{\mu^{2}}} \sqrt{\frac{q}{k_{F}}},
$$

where $C=\sqrt{g_{s} g_{v} e^{2} /\left(8 \pi \epsilon_{r} \epsilon_{0} v_{F}\right)}$ with $g_{s}$ and $g_{v}$ spinand valley degeneracies, respectively, and $\epsilon_{r}=2.5$, the relative dielectric constant for graphene on a $\mathrm{SiO}_{2}$ substrate. In the general case the plasmon dispersion may depend on the direction of the wave vector q. However, as can be seen in Fig. 3 (bottom panel), at large values of $\omega / t$ - which determine the undamped plasmons because $\operatorname{Im} \chi_{0}^{r}$ vanishes there - the azimuthal dependence is very weak, and can be neglected. This result also follows analytically from the definition of $\chi_{0}^{r}$, Eq.(5): in the small $q a$ limit it is possible to neglect the small energy difference $\epsilon_{n \mathbf{k}}-\epsilon_{n^{\prime} \mathbf{k}^{\prime}}$ as compared to $\omega$, and the azimuth-independent $1 / \omega$-behavior of $\operatorname{Re} \chi_{0}^{r}$ follows, as seen in the numerics of Fig. 3, bottom panel. In Figs. [5 
and 6 we compare the plasmon dispersion laws for pristine graphene within the Dirac cone approximation, a gapped graphene with full $\phi(\mathbf{k})$, and the $\{12,3\}$ GAL. Qualitatively, the GAL plasmon dispersion always lies below the other models. At low chemical potential the gapped graphene plasmon dispersion is essentially identical with the full calculation (Fig. (5), while for a higher $\mu$ (Fig. 6) the gapped plasmon model works worse, as expected.

\section{CONCLUSIONS}

We have analyzed screening in graphene antidot lattices (GAL) within the random phase approximation. A general procedure for calculating the polarization function is outlined. An efficient long wavelength approximation is introduced, which eases the numerical task considerably, and the accuracy of this approximation is analyzed in terms of numerical examples. We also consider another phenomenological model: gapped graphene. We have chosen a $\{12,3\}$ GAL as a generic system, and present results for this structure evaluated within the various approximation schemes. We conclude that the gapped graphene model works reasonably well for low chemical potentials $(\mu / t \simeq 0.05)$, but that it fails even qualitatively for high chemical potentials $(\mu / t \simeq 0.5)$. We also determine the undamped plasmons for the $\{12,3\}$ GAL. The plasmon dispersion law for the GAL has the same square-root behavior as pristine graphene, but it is significantly suppressed. The gapped graphene model works very well for low dopings, because the highenergy tails of the real part of the polarization are largely model independent. Future issues that need to be addressed include the effects due to the GAL symmetry, and the geometry and edge structure of the nanoperforations defining the antidots.

\section{Acknowledgments}

APJ is grateful to the FiDiPro program of the Academy of Finland.

Note added in proof. During the technical processing of this manuscript we became aware of very recent work by Scholz and Schliemann ${ }^{35}$, who give a very detailed account of the current-current correlation function of gapped graphene. Our results agree with theirs, whenever there is overlap.
1 T. G. Pedersen, C. Flindt, J. Pedersen, N. A. Mortensen, A. P. Jauho, and K. Pedersen, Phys. Rev. Lett. 100, 136804 (2008).

2 C.-H. Park, L. Yang, Y. W. Son, M. L. Cohen, and S. G. Louie, Nature Physics 4, 213 (2008).

3 C. X. Bai and X. D. Zhang, Phys. Rev. B 76, 075430 (2007).

4 M. Barbier, F. M. Peeters, P. Vasilopoulos, and J. M. Pereira, Phys. Rev. B 77, 115446 (2008).

5 A. Isacsson, L. M. Jonsson, J. M. Kinaret, and M. Jonson, Phys. Rev. B 77, 035423 (2008).

${ }^{6}$ R. Balog, B. Jorgensen, L. Nilsson, M. Andersen, E. Rienks, M. Bianchi, M. Fanetti, E. Laegsgaard, A. Baraldi, S. Lizzit, et al., Nature Mat. 9, 315 (2010).

7 L. Brey and H. A. Fertig, Phys. Rev. Lett 103, 046809 (2009).

8 T. G. Pedersen, C. Flindt, J. Pedersen, A. P. Jauho, N. A. Mortensen, and K. Pedersen, Phys. Rev. B 77, 245431 (2008).

9 T. G. Pedersen, A. P. Jauho, and K. Pedersen, Phys. Rev. B 79, 113406 (2009).

10 J. A. Furst, J. G. Pedersen, C. Flindt, N. A. Mortensen, M. Brandbyge, T. G. Pedersen, and A.-P. Jauho, New Journal of Physics 11, 095020 (2009).

11 M. Vanevic, V. M. Stojanovic, and M. Kindermann, Phys. Rev. B 80, 045410 (2009).

12 N. Vukmirovic, V. M. Stojanovic, and M. Vanevic, Phys. Rev. B 81, 041408 (2010).

13 V. M. Stojanovic, N. Vukmirovic, and C. Bruder, Phys. Rev. B 82, 165410 (2010).

14 M. Wimmer, A. R. Akhmerov, and F. Guinea, Phys. Rev. B 82, 045409 (2010).
15 W. Liu, Z. F. Wang, Q. W. Shi, J. Yang, and F. Liu, Phys. Rev. B 80, 233405 (2009).

16 J. Eroms and D. Weiss, New Journal of Physics 11, 095021 (2009).

17 J. W. Bai, X. Zhong, S. Jiang, Y. Huang, and X. Duan, Nature Nanotech 5, 190 (2010).

18 M. Kim, N. S. Safron, E. Han, M. S. Arnold, and P. Gopalan, Nano Letters 10, 1135 (2010).

19 M. Begliarbekov, O. Sul, J. Santanello, N. Ai, X. Zhang, E. H. Yang, and S. Strauf, Nano Letters 11, 1254 (2011).

20 J. A. Furst, T. G. Pedersen, M. Brandbyge, and A. P. Jauho, Phys. Rev. B 80, 115117 (2009).

21 K. W.-K. Shung, Phys. Rev. B 34, 979 (1986).

22 J. Gonzalez, F. Guinea, and M. A. H. Vozmediano, Phys. Rev. B 59, R2474 (1999).

23 B. Wunsch, T. Stauber, F. Sols, and F. Guinea, New Journal of Physics 8, 318 (2006).

24 E. H. Hwang and S. Das Sarma, Phys. Rev. B 75, 205418 (2007)

25 T. Stauber, J. Schliemann, and N. M. R. Peres, Phys. Rev. B 81, 085409 (2010).

26 T. Stauber, Phys. Rev. B 82, 201404 (2010).

27 S. H. Abedinpour, G. Vignale, A. Principi, M. Polini, W. K. Tse, and A. H. Macdonald, arXiv:1101.4291 (2011).

28 H. Bruus and K. Flensberg, Many-Body Quantum Theory in Condensed Matter Physics (Oxford University Press, 2004).

29 R. Petersen, T. G. Pedersen, and A. P. Jauho, ACS Nano 5, 523 (2011).

${ }^{30}$ F. Ouyang, S. Peng, Z. Liu, and Z. Liu, ACS Nano 5, 4023 (2011).

31 T. Tudorovskiy and S. A. Mikhailov, Phys. Rev. B 82, 
$073411(2010)$

32 O. Pulci, B. Adolph, U. Grossner, and F. Bechstedt, Phys. Rev. B 58, 4721 (1998).

33 J. A. Li, I. Martin, M. Büttiker, and A. F. Morpurgo, Nature Physics 7, 83 (2011).
34 P. K. Pyatkovskiy, J. Phys: Cond. Matter 21, 025506 (2009).

35 A. Scholz and J. Schliemann, Phys. Rev. B 83, 235409 (2011). 